\title{
On the limits of digital video microscopy
}

\author{
J. Baumgartl and C. Bechinger \\ 2. Physikalisches Institut - Pfaffenwaldring 57, 70550 Stuttgart, Germany
}

PACS. 82.70.Dd - Colloids.

PACS. 05.40.-a - Fluctuation phenomena, random processes, noise, and Brownian motion.

PACS. 61.20.-p - Structure of liquids.

\begin{abstract}
We explore the limits of digital video microscopy which is established as a standard method in physics, chemistry and biology. At particle distances close to contact we observe small but systematic deviations between the optically measured and the real particle distances. This difference is caused by the overlap of the optical images between neighboring particles. Exemplarily we discuss the consequences of this effect on pair potential measurements of charge stabilized colloids in confined geometries.
\end{abstract}

During recent years, the interest in experimental studies with micron-sized colloidal particles which are suspended in liquids is strongly increasing. Driven by Brownian motion, such particles rapidly sample their accessible configurational space thus making them dedicated for real time "simulations" under conditions which are not easily accessible with numerical methods [1-5]. Another motivation for using colloids is founded on their use as mesoscopic handles which can be attached to nanometer-sized objects. Since the position of colloidal particles can be conveniently controlled with laser optical tweezers, such tethering experiments allow to study, e.g., mechanical properties on a single molecule level $[6,7]$. In many of the above experiments positional information of colloidal spheres is obtained by digital video microscopy which is capable to resolve particle positions with sub pixel resolution down to about $10 \mathrm{~nm}$ (for a review see, e.g., [8]). However, despite the general importance and the widespread use of this technique, only few studies on the limitations of this method exist. This is particularly true for particle distances close to contact where the optical images of the colloids start to overlap. In this regime experimental investigations are mandatory because earlier calculations based on simple reflection algorithms predict a systematic overestimation of the particle distances near contact by about $80 \mathrm{~nm}[9]$.

In this letter we investigate the accuracy to which particle distances close to contact can be extracted with digital video microscopy. Our analysis shows that due to minute optical distortions caused by overlapping particles images, systematic deviations $\Delta r$ between the measured and the true particle distance occur. Since $\Delta r$ changes sign as a function of the particle distance, this effect can pretend attractive components in the pair potential of entirely repulsive systems. Therefore, our findings presented in this letter may resolve the ongoing controversial debate on the apparent long-ranged attraction of likely charged colloids under confinement [10-12]. 


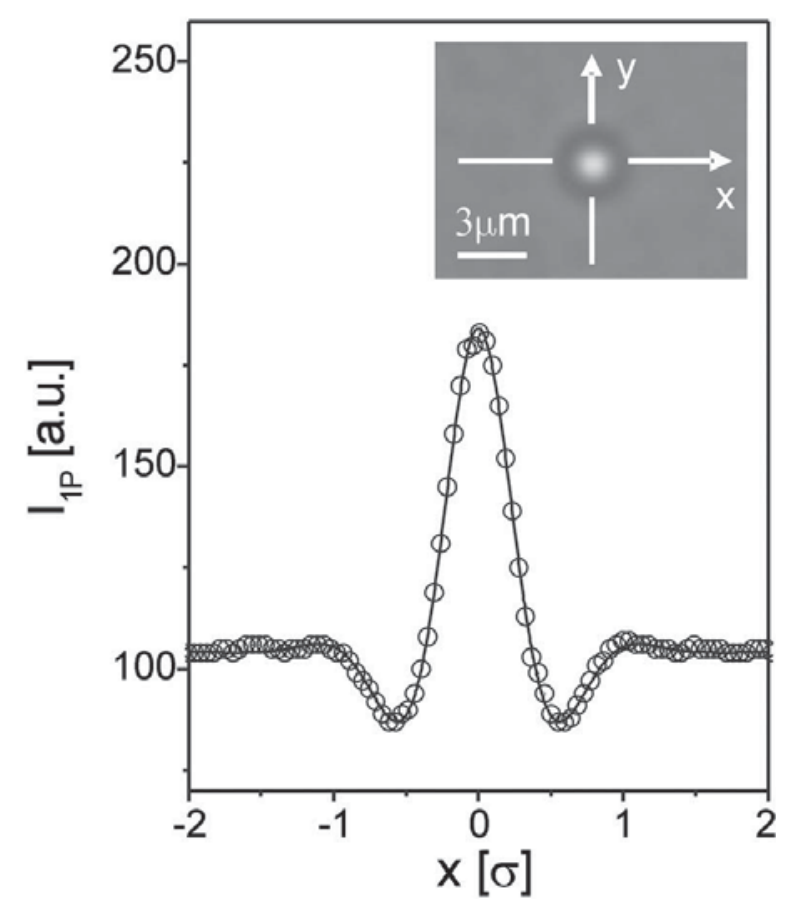

Fig. 1 - Cross-section of the intensity distribution of a transparent colloidal particle derived from the image in the inset. Experimental data (symbols) and arbitrary analytical fit function (solid line). Inset: typical snapshot of a colloidal particle observed by video microscopy.

Our measurements were performed with aqueous suspensions of highly charged, monodisperse silica spheres with diameter $\sigma=1.5 \mu \mathrm{m}$ which were confined between a cover slip and a microscope slide. A well-defined distance $d$ between the plates was achieved by adding a small amount of polystyrene particles $(\sigma=1.96 \mu \mathrm{m})$ to the suspension which act as immobile spacer particles. After confining the suspension, the cell is sealed with epoxy resin which yields stable conditions over several months. The silica spheres have been demonstrated to form a monolayer with out-of-plane excursions on the order of less than $100 \mathrm{~nm}$ [13]. For details of the sample preparation we refer to [11]. The particles were illuminated with an incoherent white-light source from above under Köhler conditions. For the imaging we used an inverted optical microscope equipped with an oil immersion objective $(100 \times$, N.A. $=1.25)$ and a CCD camera. For further analysis those images were digitized with a frame grabber (8-bit gray-scale resolution) and stored on the hard drive of a computer.

The inset of fig. 1 shows a typical snapshot of a transparent colloidal particle under bright field illuminating conditions. The bright central spot and the darker ring around the particles are typical for images of transparent colloids and are in good agreement with the results of other authors $[11,12,14]$. From the digitized intensity distribution we calculated a cross-section of the intensity distribution (symbols in fig. 1). The solid line is an (arbitrary) analytical expression $I(x)$ which was chosen to yield best agreement with our experimental data. As can be seen, the intensity has a maximum at the particle center and rapidly decays in radial direction. It is important to realize that before the intensity reaches a constant background level it falls below this value around $x \approx 0.5$. When comparing the spatial extension of the intensity distribution with the geometrical size of the particle, it is also obvious that the particle image is blurred over almost twice the sphere diameter $\sigma$. Due to the radial symmetry of the problem, from $I(x)$ one easily obtains the corresponding two-dimensional (2D) one-particle intensity distribution $I_{1 P}(x, y)$. Both, the undershooting of the intensity below the background and 


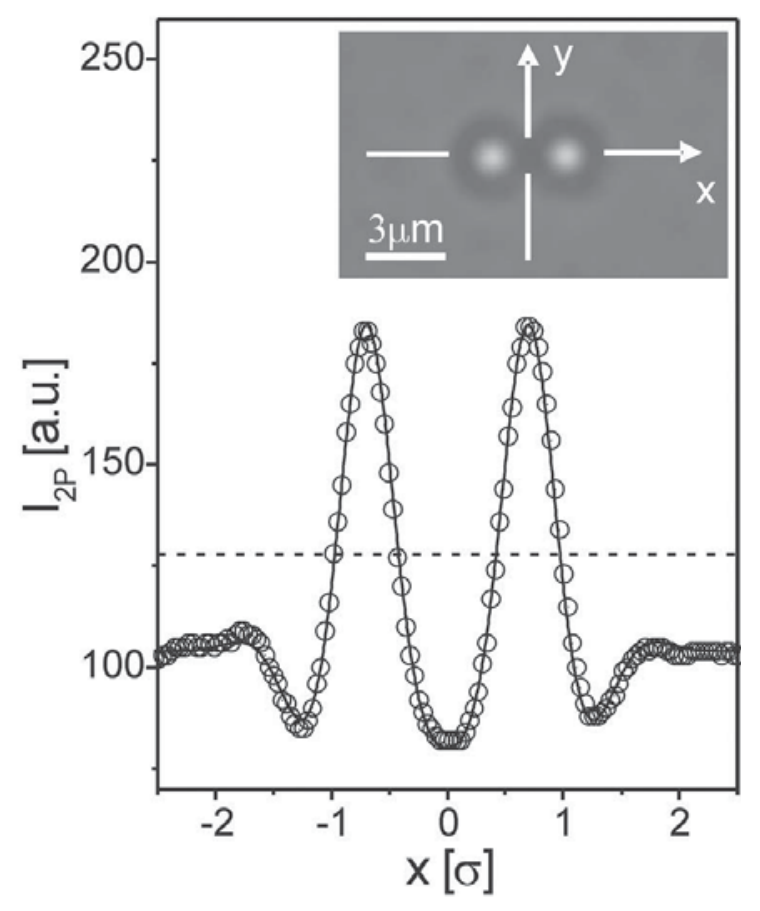

Fig. 2 - Cross-section of the intensity distribution of two colloids close to contact. Experimental data (symbols) and linear superposition of the analytical one-particle intensity function (solid line) from fig. 1. The dashed line indicates the threshold value applied to the image processing algorithm for extracting the measured center-to-center particle distance from optical images. Inset: snapshot of two colloidal particles obtained by video microscopy.

the (compared to $\sigma$ ) enlarged optical image of the particles are in qualitative agreement with calculations of Ovryn who made a complete numerical analysis of the imaging of transparent colloidal spheres. By means of Mie theory calculations he computed the electromagnetic field scattered by a spherical particle under plane-wave illumination conditions and how it is imaged by a high numerical aperture microscope objective $[15,16]$.

The open symbols in fig. 2 correspond to the intensity cross-section taken from a snapshot (inset of fig. 2) of two silica particles close to contact. In contrast to fig. 1, in the presence of a second colloidal sphere the intensity distribution becomes slightly asymmetric around the particle centers. This is different compared to fig. 1. Assuming that the optical image of a particle pair follows from a linear superposition of single-particle images (incoherent image formation) the intensity distribution of two colloids with center-to-center distance $r$ is given by $I_{2 P}(x, y ; r)=I_{1 P}(x-r / 2, y)+I_{1 P}(x+r / 2, y)-I_{0}$, where $I_{0}$ accounts for the background intensity. The solid line in fig. 2 corresponds to the cross-section $I_{2 P}(x, y=0 ; r)$. The excellent agreement with the experimental data clearly demonstrates that under our illumination conditions a linear superposition yields accurate results. Having obtained an analytical expression for $I_{2 P}$ we now can calculate optical images for different particle distances $r$. From these images we obtain the optically determined particle center distances $r_{m}$ by applying a standard image processing algorithm. After subtraction of a uniform threshold $T$ we calculate the intensity weighted centroids of the two particles within the areas defined by $I_{2 P}(x, y ; r) \geq T[8]$. The threshold $T$ is indicated by the dashed line in fig. 2 . From this we obtain the difference between the (via digital video microscopy) measured and the true particle distance $2 \Delta r=r_{m}-r$. The results are plotted in fig. 3 as solid line vs. $r_{m}$ and show a small but systematic signature which we also observed for other particle sizes. For 


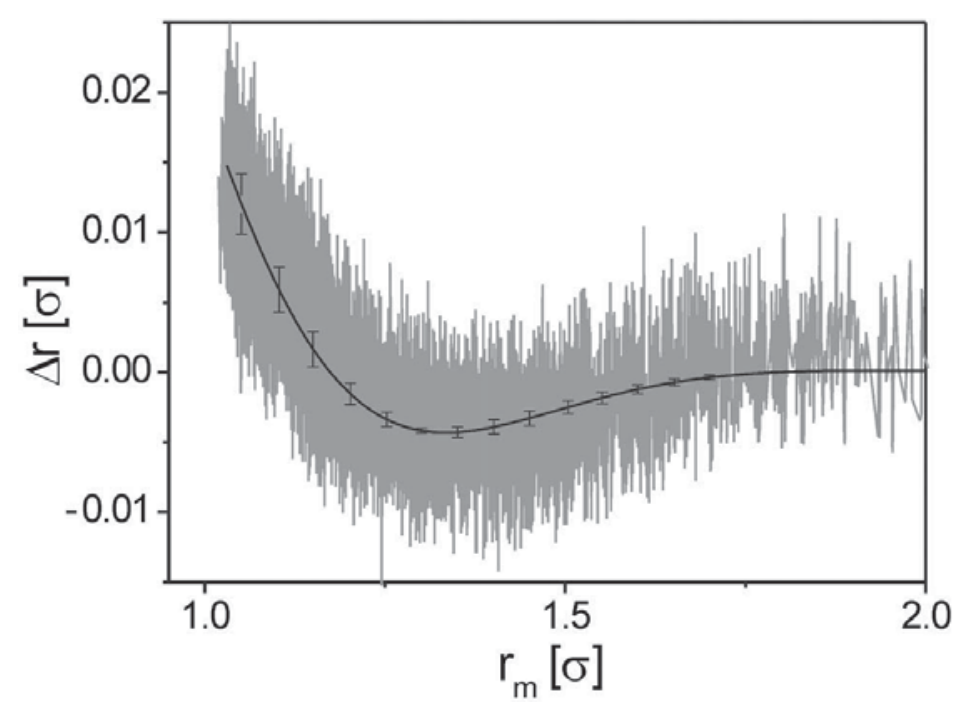

Fig. $3-\Delta r$ vs. $r_{m}$ determined numerically (black solid line) and experimentally (grey solid line). The error bars indicated the change of $\Delta r$ upon variation of the applied intensity threshold for particle detection by $\pm 10 \%$.

$1 \leq r_{m} \leq 1.15$ the measured distance overestimates the real particle distance while the opposite is the case for $1.15 \leq r_{m} \leq 1.8$. This change in sign is in contrast to earlier calculations where the spheres images were modeled with parabolic caps which then results in a monotonic behavior of $\Delta r\left(r_{m}\right)$ [9]. The error bars in fig. 3 denote the change of $\Delta r$ upon variation of the applied intensity threshold $T$ by $\pm 10 \%$ and indicate that the threshold value has (within certain limits) only a small influence on $\Delta r$.

In order to compare the calculated particle images in fig. 2 with experimental data, we performed accurate measurements of the center-to-center distance distribution between particle pairs in a highly diluted colloidal system. With the above-described image processing algorithm we first determined the position of an isolated colloid which was irreversibly bound to the substrate to act as an immobile positional reference particle (due to van-der-Waals forces one always observes some of our particles stuck to the glass surfaces). Due to randomly distributed digitization noise in the CCD camera and the frame grabber which is unavoidable in video microscopy, the time-averaged measured particle position distribution is a Gaussian with a half-width of $15 \mathrm{~nm}$ (corresponding to our experimental resolution). The center of this distribution is identified as the true reference particle position $\left(\bar{x}_{r e f}, \bar{y}_{r e f}\right)$. Then a second free colloid was confined in the vicinity of the reference particle by an optical laser trap. In this trap, which was formed by a slightly defocused, vertical incident Gaussian laser beam $\left(\mathrm{TEM}_{00}\right.$, $\lambda=532 \mathrm{~nm}$ ) with a half-width of about $4 \mu \mathrm{m}$ and a power of $50 \mathrm{~mW}$, the sphere performed in-plane fluctuations owing to the Brownian motion. From the recorded images we determined the time-dependent position of the fluctuating particle $\left(x_{\text {free }}(t), y_{\text {free }}(t)\right)$ in the laser trap. From this we obtain $\Delta r(t)=r_{m}(t)-\sqrt{\left[x_{\text {free }}(t)-\bar{x}_{r e f}\right]^{2}+\left[y_{\text {free }}(t)-\bar{y}_{r e f}\right]^{2}}$ which is plotted vs. $r_{m}$ in fig. 3 as a grey curve. Since we have only knowledge about the true position of one, namely the reference particle, the factor two on the left side of the equation is missing here. The data points which scatter within our experimental resolution are in excellent agreement with the above results obtained from calculated particle images and show the same systematic deviation between the via video microscopy measured and the real particle distance. We want to emphasize that all parameters for the image processing (in particular the threshold value $T$ ) were identical for the experimentally and the numerically obtained intensity patterns. 


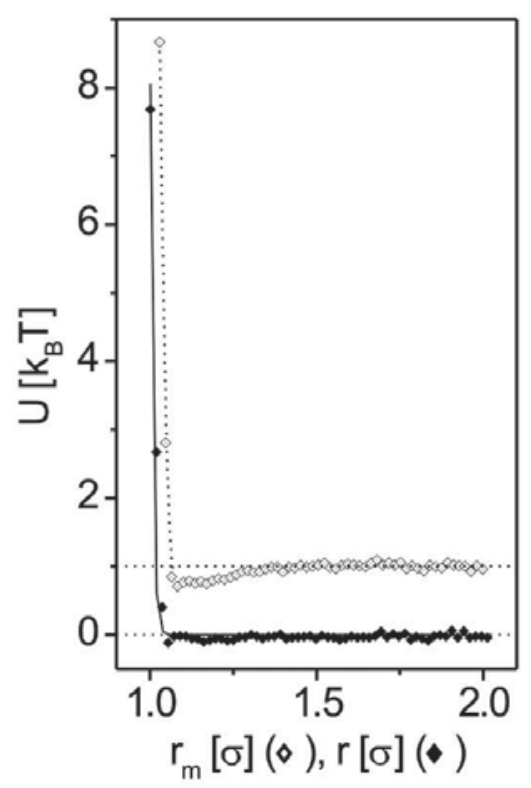

Fig. 4 - Measured pair potentials vs. the measured (open symbols) and the corrected (closed symbols) particle distance of two colloidal particles in confined geometry obtained by video microscopy. The data are shifted in vertical direction for clarity. The solid line corresponds to a least-mean-square fit to a screened Coulomb potential with fit parameters $Z^{*} \approx 8000 \pm 4000$ and $\kappa \approx 0.1 \mathrm{~nm}^{-1}$. The dotted lines are guides to the eye.

At first glance, the deviation between the measured and the true particle distance seems to be rather small (about 1\% of the particle diameter) and it appears questionable whether such a tiny effect may have a noticeable influence on experimental results. In order to demonstrate, however, that even small deviations $\Delta r$ can indeed pretend sizable effects, we measured the pair potential of two free colloidal particles which were both confined in the above-described laser trap. From the measured distance distribution $P_{m}\left(r_{m}\right)$ we extracted the pair potential $U\left(r_{m}\right)$ via $P_{m}\left(r_{m}\right)=P_{0} \exp \left[-\left(U_{\text {ext }}^{1}+U_{\text {ext }}^{2}+U\left(r_{m}\right)\right) / k_{B} T\right]$ with $U_{\text {ext }}^{1,2}$ accounting for the potential energy of the two particles induced by the optical laser field. In the central region which is sampled by the particles, we confirmed that $U_{e x t}^{1,2}$ is to good approximation a parabolic potential. Under these conditions the total energy is given by the sum of a contribution depending on the particle distance and one depending only on the center of mass of the colloidal pair. This allows one to obtain $U_{e x t}^{1,2}$ and $U\left(r_{m}\right)$ in the same experiment (in case of non-parabolic light traps the external potential must be first determined by the probability distribution of a single colloidal particle inside the laser trap [17]). When plotting the resulting pair potential vs. the uncorrected distance $r_{m}$ (open symbols in fig. 4) a shallow minimum around $1.2 \sigma$ with a depth of about $0.25 k_{B} T$ is observed. In order to demonstrate the effect of the optical artifact on the pair potential, from the measured distance distribution $P_{m}\left(r_{m}\right)$ we first calculate the corresponding corrected distribution $P(r)=P_{m}\left(r_{m}\right) \frac{\mathrm{d} r_{m}}{\mathrm{~d} r}$. Since $\frac{\mathrm{d} r_{m}}{\mathrm{~d} r}$ depends on the particle distance $r_{m}$ (see fig. 3) this leads to a nonlinear correction of $P(r)$ and thus to a qualitative change in the true pair potential which is plotted in fig. 4 as closed symbols (for clarity the data are shifted in vertical direction by $1 k_{B} T$ ). The most striking feature of the correction is the disappearance of the minimum compared to the uncorrected pair potential.

Long-ranged attractive components in the pair potential at distances $1.2 \sigma<r_{m}<1.5 \sigma$ and strengths of several tenths $k_{B} T$ (similar to the uncorrected potential in fig. 4) were reported by several groups when measuring the pair potential of charge-stabilized colloidal 
particles in confined geometries $(d<20 \mu \mathrm{m})$ [10-12,18]. This so-called like-charge attraction (LCA) provoked a controversial and still ongoing discussion in colloidal science because it challenges our current understanding of colloidal interactions. Despite intense theoretical and numerical studies, however, no conclusive explanation of LCA is available while at the same time mean-field theories even refute the existence of a minimum in the interaction potential of likely charged colloids independent of the specific confinement conditions $[19,20]$.

In face of the striking similarity of the uncorrected pair potential in fig. 4 and the characteristic features of LCA, we believe that the above-mentioned studies of other authors $[10-12,18]$ are contaminated by the optical artifact as described here. This interpretation is also consistent with the fact, that LCA was only observed under confinement while pair-potentials in thick samples cells are experimentally confirmed to be entirely repulsive $[17,21]$. The effective screening length in confined geometries is dominated by the counterions of the charged glass walls of the sample cell which strongly screen the pair interaction and result in particle distances below $2 \sigma$. In this regime, the particle images start to overlap and thus lead to the artifacts as reported here. This is in contrast to unconfined samples where the electrostatic screening due to counterions of the walls is much smaller and particle distances below $2 \sigma$ are hardly sampled. In this case $\Delta r \approx 0$ and video microscopy yields accurate results.

Finally, we compared the corrected pair potential with a screened Coulomb potential (solid line in fig. 4) $U(r)=\left(Z^{*}\right)^{2} \lambda_{B}\left(\frac{\exp [\kappa R]}{1+\kappa R}\right)^{2} \frac{\exp [-\kappa r]}{r}$ with $Z^{*}$ the effective colloidal charge, $R$ the particle radius, $\kappa$ the inverse Debye screening length, and $\lambda_{B}=0.72 \mathrm{~nm}$ the Bjerrum length in water. As fitting parameters we obtain $Z^{*} \approx 8000 \pm 4000$ and $\kappa \approx 0.1 \mathrm{~nm}^{-1}$. In particular, $Z^{*}$ is close the value obtained in an independent measurement for the same particles in a thicker sample cell where only particle distances above $2 \sigma$ were sampled.

It is an interesting question, to what extent the artifacts as described here are present in confocal microscopy. While the imaging process in confocal microscopy differs somewhat from conventional video microscopy, both techniques are diffraction limited and we assume that qualitatively similar artifacts occur. A possible solution to avoid these artifacts are coreshell particles with a fluorescent core [22]. For sufficiently small cores, the corresponding fluorescent images do not overlap even when the particles are at contact. Therefore we expect that under these conditions no artifacts will occur.

In summary, we have demonstrated that optical artifacts caused by overlapping images of adjacent objects can pretend slightly wrong particle distances as determined with digital video microscopy. Accordingly, if no corrections are applied, the interpretation of small particles distances has to be taken with care. This is particularly important for colloidal interaction studies in confined samples where particle distances below $2 \sigma$ are sampled. At those distances optical artifacts can pretend long-ranged attractive components.

We want to thank J.-L. Arauz-Lara and A. RAmirez-Saito for the preparation of thin sample cells, and A. RoHrBACH for helpful discussions.

\section{REFERENCES}

[1] Frenkel D., Physica A, 313 (2002) 1.

[2] Kegel W. K. and Blaaderen A. V., Science, 287 (2000) 290.

[3] Yethiraj A. and Blaaderen A. V., Nature, 421 (2003) 513.

[4] Poon W., Pusey P. and Lekkerkerker H., Phys. World, April issue (1996) 27.

[5] Babic D. and Bechinger C., Phys. Rev. Lett., 94 (2005) 148303. 
[6] Sheetz M. P., in Laser Tweezers in Cell Biology, Vol. 55 (Academic Press) 1998.

[7] Jiang G., Giannone G., Critchley D. R., Fukumoto E. and Sheetz M. P., Nature, 424 (2003) 334 .

[8] Crocker J. C. and Grier D. G., J. Colloid Interface Sci., 179 (1996) 298.

[9] Verma R., Crocker J. C., Lubensky T. C. and Yodh A. G., Macromolecules, 33 (2000) 177.

[10] Kepler G. M. and Fraden S., Phys. Rev. Lett., 73 (1994) 356.

[11] León G. C. D., Saucedo-Solorio J. M. and Arauz-Lara J. L., Phys. Rev. Lett., 81 (1998) 1122.

[12] Han Y. and Grier D. G., Phys. Rev. Lett., 91 (2003) 038302.

[13] Behrens S. H. and Grier D. G., Phys. Rev. E, 64 (2001) 50401.

[14] Baksh M. M., Jaros M. and Groves J. T., Nature, 427 (2004) 139.

[15] Ovryn B. and Izen S. H., J. Opt. Soc. Am. A, 17 (2000) 1202.

[16] Ovryn B., Exp. Fluids, 29 (2000) S175.

[17] Brunner M., Dobnikar J., Grünberg H. H. v. and Bechinger C., Phys. Rev. Lett., 92 (2004) 078301.

[18] Grier D. G. and Han Y., J. Phys. Condens. Matter, 16 (2004) S4145.

[19] Trizac E. and Raimbault J.-L., Phys. Rev. E, 60 (1999) 6530.

[20] Sader J. E. and Chan D. Y. C., Langmuir, 16 (2000) 324.

[21] Vondermassen K., Bongers J., Mueller A. and Versmold H., Langmuir, 10 (1994) 1351.

[22] Blaaderen A. V. and Wiltzius P., Science, 287 (1995) 290. 